Article

\title{
Vaccine Design from the Ensemble of Surface Glycoprotein Epitopes of SARS-CoV-2: An Immunoinformatics Approach
}

\author{
Noor Rahman ${ }^{1}\left(\mathbb{0}\right.$, Fawad Ali $^{2}{ }^{\circledR}$, Zarrin Basharat ${ }^{3}{ }^{(0)}$, Muhammad Shehroz $^{4}$, \\ Muhammad Kazim Khan ${ }^{5}$, Philippe Jeandet ${ }^{6} \mathbb{D}$, Eugenie Nepovimova ${ }^{7}$, Kamil Kuca ${ }^{7, *(\mathbb{D})}$ and \\ Haroon Khan $8, *$ (D) \\ 1 H.E.J. Research Institute of Chemistry, International Center for Chemical and Biological Sciences, \\ University of Karachi, Karachi 75270, Pakistan; noorbiochemist@gmail.com \\ 2 Department of Biochemistry, Hazara University, Mansehra 21120, Pakistan; fawwadali792@gmail.com \\ 3 Jamil-ur-Rahman Center for Genome Research, PCMD, ICCBS, University of Karachi, \\ Karachi 75270, Pakistan; zarrin.iiui@gmail.com \\ 4 Department of Biotechnology, Virtual University of Pakistan, Lahore 54000, Pakistan; \\ muhammad.shehroz@vu.edu.pk \\ 5 Centre for Applied Molecular Biology, University of the Punjab, Lahore 53700, Pakistan; kazimbt@gmail.com \\ 6 Faculty of Sciences, University of Reims Champagne-Ardenne, CEDEX 2, 51687 Reims, France; \\ philippe.jeandet@univ-reims.fr \\ 7 Department of Chemistry, Faculty of Science, University of Hradec Kralove, \\ 50005 Hradec Kralove, Czech Republic; eugenie.nepovimova@uhk.cz \\ 8 Department of Pharmacy, Abdul Wali Khan University Mardan, Mardan 23200, Pakistan \\ * Correspondence: kamil.kuca@uhk.cz (K.K.); haroonkhan@awkum.edu.pk (H.K.); \\ Tel.: +420-603-289-166 (K.K.); +92-332-9123171 (H.K.)
}

Received: 7 June 2020; Accepted: 20 July 2020; Published: 28 July 2020

\begin{abstract}
The present study aimed to work out a peptide-based multi-epitope vaccine against the severe acute respiratory syndrome coronavirus 2 (SARS-CoV-2). We predicted different B-cell and T-cell epitopes by using the Immune Epitopes Database (IEDB). Homology modeling of the construct was done using SWISS-MODEL and then docked with different toll-like-receptors (TLR4, TLR7, and TLR8) using PatchDock, HADDOCK, and FireDock, respectively. From the overlapped epitopes, we designed five vaccine constructs $\mathrm{C} 1-\mathrm{C} 5$. Based on antigenicity, allergenicity, solubility, different physiochemical properties, and molecular docking scores, we selected the vaccine construct 1 (C1) for further processing. Docking of C1 with TLR4, TLR7, and TLR8 showed striking interactions with global binding energy of $-43.48,-65.88$, and $-60.24 \mathrm{Kcal} / \mathrm{mol}$, respectively. The docked complex was further simulated, which revealed that both molecules remain stable with minimum RMSF. Activation of TLRs induces downstream pathways to produce pro-inflammatory cytokines against viruses and immune system simulation shows enhanced antibody production after the booster dose. In conclusion, $\mathrm{C} 1$ was the best vaccine candidate among all designed constructs to elicit an immune response SARS-CoV-2 and combat the coronavirus disease (COVID-19).
\end{abstract}

Keywords: vaccine; multi-epitopes; antigenicity; allergenicity; coronavirus; pneumonia

\section{Introduction}

The 2019 SARS-like coronavirus (SARS-CoV-2), a member of betacoronavirus, emerged in late December 2019, causing pneumonia [1]. Coronaviruses are enveloped, large, positive-sense RNA viruses belonging to the family of Coronaviridae, that can infect mammals, birds, and humans, causing deadly 
pneumonia [2]. The Coronaviridae family consists of two subfamilies (1) Coronaviridae, which contain the genera alpha, beta, gamma, and deltacoronavirus and (2) Torovirinae, which comprise a single genera Torovirus as well as unknown genera [3].

Since the start of the twenty-first century, two beta coronaviruses have caused deadly pneumonia in humans. In 2002-2003, the severe acute respiratory syndrome coronavirus (SARS-CoV) emerged from being accountable for an outbreak with a death rate of $10 \%$ and spread to 5 continents. In 2012, the Middle East respiratory syndrome coronavirus (MERS-CoV) became prominent in Saudi Arabia and caused repeated outbreaks in humans with a 35\% death rate [4,5]. In December 2019, a novel SARS-like coronavirus (SARS-CoV-2) emerged, that caused pneumonia with high morbidity and mortality rates responsible for 3,303,296 infections, 235,290 deaths in 185 countries; Dated 1 May 2020 (https://gisanddata.maps.arcgis. com/apps/opsdashboard/index.html\#/bda7594740fd40299423467b48e9ecf6). SARS-CoV, MERS-CoV, and 2019-SARS-CoV-2 are zoonotic and their primary hosts are bats and civets, camel being an intermediate host. However, it was suggested that the SARS-CoV-2 was directly transferred from bats to humans from Wuhan seafood in the Hubei province of China. Other reports revealed the occurrence of human-to-human transmission [1,4,5]. There are four other coronaviruses, HCoV-229E and HCoV-NL63 (alpha coronaviruses) and HCoV-HKU1 and HCoV-OC43 (beta coronaviruses), responsible for mild respiratory tract infections that cause complications or fatalities in elderly immuno-compromised individuals and young children [6]. Currently, there are some antiviral treatments like Chloroquine, Remdesivir, Ribavirin, Lopinavir, traditional Chinese medicine etc., under trial to combat SARS-CoV-2. In silico drug screening against viral proteins using natural products, FDA approved drugs, and other antivirals have also been attempted [7]. A whooping 657 clinical trials for various drugs against COVID-19 have been registered till 20 April 2020 (https://clinicaltrials.gov/ct2/results?cond=COVID-19). Although some studies report the use of these drugs in some COVID-19 patients, their concordant status against the disease is yet inconclusive and no satisfactory treatment for COVID-19 exists till to date. It has been proposed that sequence similarity and computational approaches can lead to vaccine design, based on derived epitope and antigen information from surface glycoprotein of SARS-CoV-2 [7]. It has also been proposed that the initial antigenic target of vaccine should be the surface protein since it allows entry of coronavirus into the host [8]. Yuan et al. (2020) [9] isolated a neutralizing antibody, bound to surface protein of a COVID-19 patient, which provides evidence that spike protein produces an antibody response and could be an effective target for vaccine design.

The S viral protein of the SARS-CoV-2 is a single polypeptide chain of 1273 amino acids [1]. The surface (S) glycoprotein of coronavirus showed in Figure 1 contains a homotrimer which is used for virus entry to promote host attachment and fusion of the virus with host cell membranes [7]. Surface glycoprotein is a class I viral fusion protein which represents the leading focal point for vaccine designing as it is the principal antigen of the virus that neutralizes antibodies during infection [2]. It contains two subunits, S1 and S2, generated by the action of host proteases which are bound by intermolecular forces in the pre-fusion conformation. The N-terminal S1 ectodomain is composed of four beta-rich domains, designated as A, B, C, and D with A or B comprises a receptor-bounding domain.

The C-terminal S2 subunit is a transmembrane domain that mediates membrane fusion [10,11]. Currently, no registered vaccine is available for COVID-19, but four vaccines are under trial while many more are under development. Computational epitope mapping is a swift way to add to the knowledge base of the COVID-19 vaccine landscape. Epitopes are the antigenic determinants, located in proteins that have the capability of initiating a cellular immune response, which in turn is arbitrated by $\mathrm{T}$ or $\mathrm{B}$ cells. T cell epitopes are usually protein antigen-derived peptides presented by major histocompatibility complex (MHC) molecules on antigen-presenting cells. These are recognized by T-cell receptors and hence, called T-cell epitopes. B cell epitopes are capable of binding an antibody. Both of these cells mediate adaptive immunity i.e., develop pathogen-specific memory. This convenes immunological protection. Identification and study of epitopes are therefore of prime interest to scientists working on diagnostic assay development and epitope-based vaccines. In silico prediction of epitopes is a swift and economical method that helps sift useful candidates from an ensemble of predicted ones. We adopted 
a multi-epitope-based strategy for this study due to several benefits of multi epitope-based vaccine design, including rational engineering of the epitopes for better potency, improved safety profiling, and spotlighting of immune responses for conserved epitopes [12]. A chemoinformatics approach for the development of a multi-epitope vaccine, as well as it's in silico cloning and expression against the deadly COVID-19, could provide clues to combat human coronavirus.

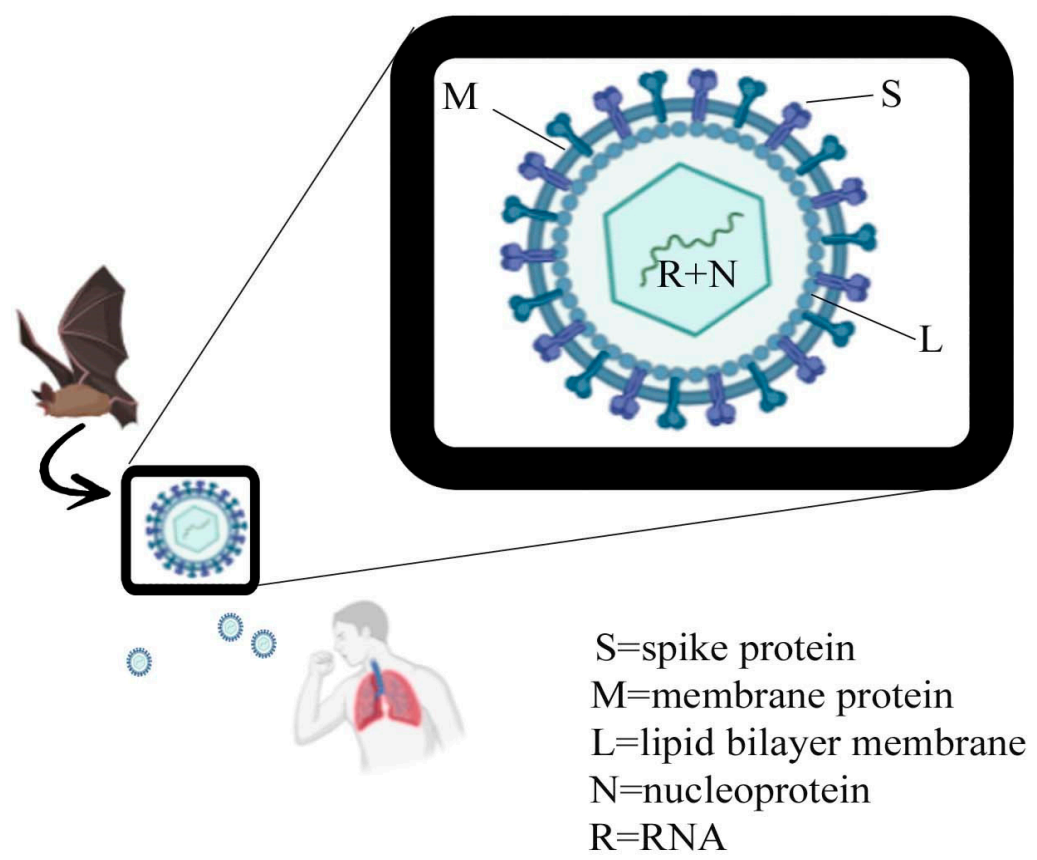

Figure 1. Different proteins and RNA + Nucleoprotein are shown in the structure of the novel SARS-like coronavirus (SARS-CoV-2), originated from the bat and causative agent of COVID-19.

\section{Materials and Methods}

\subsection{Protein Sequence Retrieval}

In the current strategy, the art of immunoinformatics approach was used for the development of the multi-epitope-based vaccine. The stepwise analysis followed for the study is depicted in Figure 2. The amino acid sequence (1273 amino acids) of the surface glycoprotein SARS-CoV-2 was retrieved from NCBI (Accession no: AYN64561.1) (https://www.ncbi.nlm.nih.gov/).

\subsection{Epitope Prediction}

For chimeric vaccine development, the surface topology of the glycoprotein was selected to identify immunogenic determinants for the vaccine construct. Identification of immunogenic T-Cells (MHC-I and II) was carried out by using the Immune Epitopes Database (IEDB) (https://www.iedb.org/) [13]. It employs a different prediction method for MHC epitope binding analysis. MHC class I and II molecules were predicted through the Stabilized Matrix Method (SMM) scoring and neutral-network based tool (net MHC-1.1) [14-16]. Epitopes were prioritized from the predicted ensemble, based on a threshold value of $\mathrm{IC}_{50} \leq 200 \mathrm{nM}$. B-Cell immunogenic determinants were projected using BCPRED (http://ailab.cs.iastate.edu/bcpreds/), with the default parameters. BCpred predicts linear B-Cell epitopes [17], which are important for stimulating a humoral immune response, which activates B lymphocytes for antibody production [18]. Interferon-inducing epitopes were identified from the MHC-II binding epitopes by using the IFNepitope web server (http://crdd.osdd.net/raghava/ifnepitope/ predict.php). IFNepitope predicts regions in the protein sequence or antigen, that cause induction of Interferon-gamma (IFN-gamma) (Dhanda et al., 2013). The overlapped epitopes were submitted to 
IFNepitope web server and IFN-gamma was predicted based on Support Vector Machine (SVM) and model was predicted by selecting IFN-gamma versus non-IFN-gamma [19].

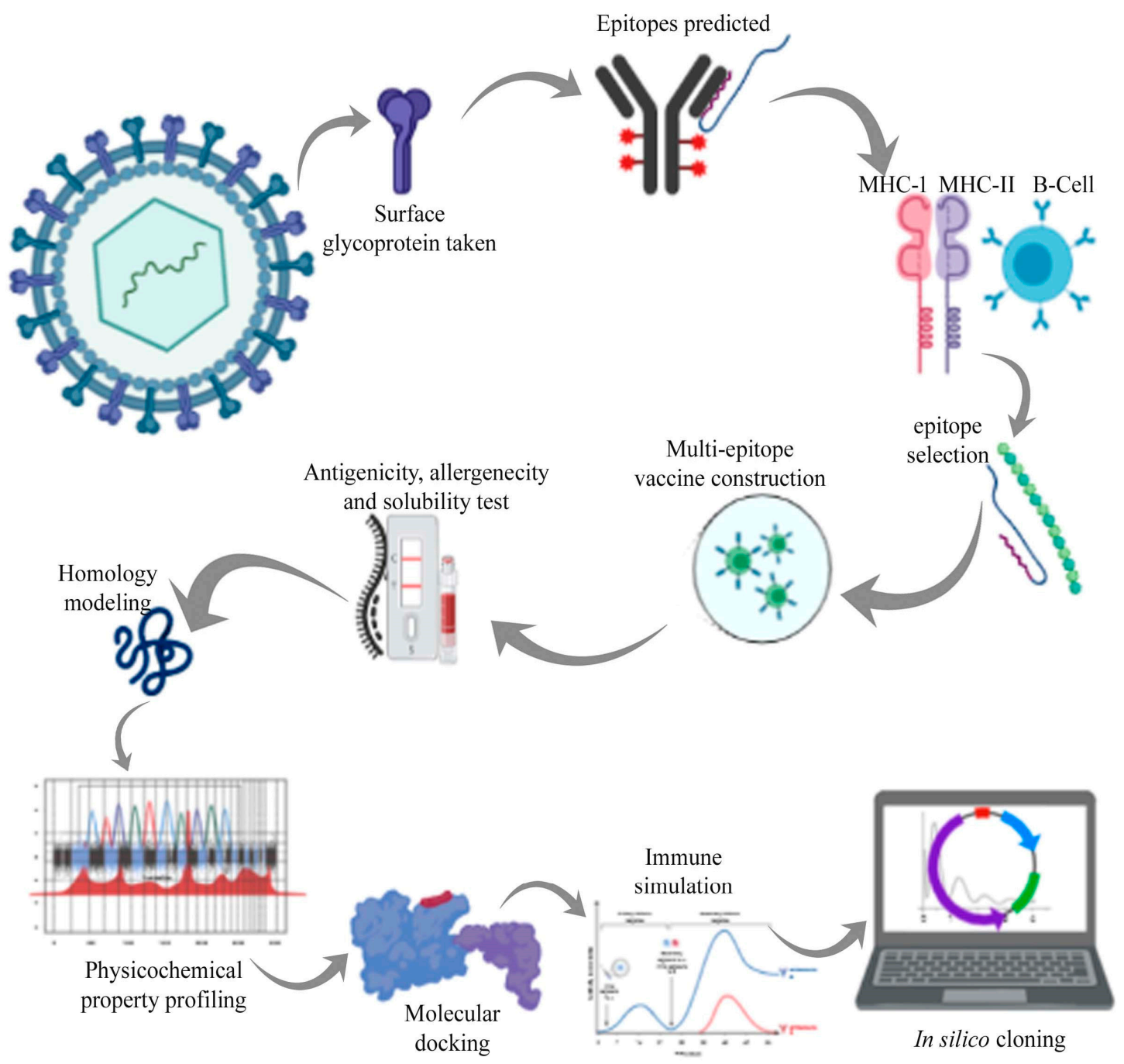

Figure 2. Schematic workflow followed for the multi-epitope vaccine design.

\subsection{Epitopes Selection and Vaccine Construction}

The B-cell and T-cell epitopes are immunodominant and crucial to enhance neutralizing-antibody response when exposed to toxins and contagions. Identification of B-cell epitopes is indispensable for epitope-based vaccine design and development [20]. MHC encodes cell surface proteins which are vital for the adaptive immune system, including MHC-II binding interferon-inducing epitopes. B-Cell, MHC-I, MHC-II, and IFN epitopes were ranked based on cut off values and compared manually for overlapped peptides. Four overlapped and high score epitopes were selected for vaccine constructs. Universal T-helper epitopes, PADRE (Pan HLA-DR reactive epitope) and five different adjuvants were added. PADRE is projected as a simple carrier epitope, useful in making synthetic or recombinant vaccines and known to have enhanced response when coupled with adjuvant [21]. Adjuvant increases the immune response of a vaccine, leading to more antibody production and reduces the quantity of antigen input. Adjuvants used in this study were mined from literature and included heparin-binding hemagglutinin (HBHA) from Mycobacterium sp., Beta defensin, Ribosomal protein, and flagellin. HBHA has been implicated as a vaccine adjuvant, with demonstrated usage in antitumor immunotherapy as well [22]. Beta defensin is an antimicrobial peptide with a role in innate immune responses and can initiate cellular immune response [23]. Ribosomal protein has been 
identified as a non-traditional adjuvant [24], while flagellin activates diverse cell types, which are part of innate and adaptive immunity, promoting cytokine production [25]. Effectiveness of all these adjuvants has been reported in the literature and they initiate cellular immune responses, leading to antigen-specific immune response stimulation. All of these are TLR agonists and it is reported that binding with TLRs shows enhanced results in activating immune responses because TLRs conduct signaling and activate the innate and adaptive immune response [26]. After addition of PADRE and the adjuvant, the vaccine constructs were admixed in different combinations with the help of GGGS, HEYGAEALERAG, and EAAAK linkers $[27,28]$.

\subsection{Determination of Antigenicity, Allergenicity, Toxicity, and Solubility}

Antigenicity of the constructed vaccine was predicted through Vaxijen v2.0 (http://www.ddgpharmfac.net/vaxijen/VaxiJen/VaxiJen.html) [29] and predicted antigenic peptides (http://imed.med. ucm.es/Tools/antigenic.pl) was used to determine the antigenicity of the predicted epitopes. An online server, AlgPred (http://crdd.osdd.net/raghava/algpred/) was used to determine the allergenicity of the constructs with -0.4 threshold value [30]. The solubility of the constructed vaccine upon expression in Escherichia coli was determined by using SOLpro (http://scratch.proteomics.ics.uci.edu/) [31].

\subsection{Physiochemical Properties}

Expasy Protparam (https://web.expasy.org/protparam/) was used to predict physicochemical properties like amino acid composition, atomic composition, molecular weight, theoretical pI, extinction coefficient, estimated half-life, aliphatic index, grand average of hydrophobicity (GRAVY), and instability index of the vaccine constructs [32].

\subsection{Homology Modeling and Model Validation}

Homology models were built for the vaccine construct, using the SWISS-MODEL (https:// swissmodel.expasy.org/) online server, which is the most reliable server for protein modeling [33]. The 3D model structure was further validated using RAMPAGE Ramachandran plot (http://mordred. bioc.cam.ac.uk/ \{\}rapper/rampage.php).

\subsection{Molecular Docking and MD Simulation}

Molecular docking is used to predict the possible binding orientation of the vaccine constructs [34, 35]. TLR7/8 complex (TLR8 PDB ID:3w3g) was downloaded from Protein Databank (PDB) (https://www. rcsb.org). Online servers PatchDock (https://bioinfo3d.cs.tau.ac.il/PatchDock/), HADDOCK webserver (https://doi.org/10.1007/978-1-4939-0366-5_12), and FireDock (http://bioinfo3d.cs.tau.ac.il/FireDock/) were used for molecular docking and docking refinement, respectively. FireDock refines and re-scoring the docked complexes and ranks the complexes based on binding score and global binding energy [36]. An online server CABS-flex (http://212.87.3.12/CABSflex2/job/7e5d9345a1a8235/) was used for molecular dynamics simulation, to check the stability of the complex.

\subsection{Immune Simulation of Vaccine Construct}

Immune simulation of vaccine construct $\mathrm{C} 1$ and positive control (construct having no adjuvant) using web server C-ImmSim (http://kraken.iac.rm.cnr.it/C-IMMSIM/) was done. It is an agent-based model implementation where information about the humoral as well as the cellular response of the mammalian immune system, invoked by antigen at the cellular level is obtained. The simulation time steps 1,42 , and $126 \mathrm{~h}(8 \mathrm{~h}$ correspond to one cell division cycle in real life according to Kaba et al. (2018)) [37] were chosen along with homozygous host haplotypes (HLA-A*0101, HLA-A*0201, HLA-B*0702, HLA-B*3901, HLA-DRB1*0101, and HLA-DRB1*0401), while other general simulation parameters were: random seed: 1234, simulated volume: 10, simulation steps 1000, Adjuvant $=100$ [38]. The comparison was drawn between positive control and construct and results were interpreted. 


\subsection{Codon Optimization and In Silico Cloning}

A codon optimization approach was used to enhance recombinant protein expression. Codon optimization is important because degeneracy of genetic code permits most of the amino acids to be encoded by multiple codons. Java codon adaptation index (JCAT) (http://www.jcat.de/) and EMBOSS Backtranseq (www.ebi.ac.uk/) were used in the codon system of $E$. coli to obtain the codon adaptation index (CAI) values and GC contents [39].

The optimized sequence of the final vaccine construct was inserted in a vector for expression, by using the Snapgene tool available at (https://www.snapgene.com/free-trial/). A compatible plasmid vector pET-28a (+) was used to integrate the optimized sequence and clone the constructed chimeric vaccine. pET-28a $(+)$ is for expression of N-terminally $6 \times$ His-tagged proteins and usually, N-terminal tags are advantageous over C-terminal tags, leading to enhanced purification, protein recovery, and stronger response.

\section{Results}

The T cell, B cell, and IFN-inducing epitopes of the antigenic surface glycoprotein were predicted with the help of Immune Epitope Database (IEDB) and IFNepitope servers. The predicted T cell epitopes were able to bind with MHC-1 and MHC-2 molecules to activate the adaptive immune response in the host. We searched the overlapped epitopes as shown in Table 1 (the epitopes bind with both MHC molecules, B cells, and IFN-gamma) to take advantage and produce humoral, cell-mediated immune responses and induce interferons which were our ultimate objective.

Table 1. Overlapped (Red) epitope of B-cell, MHC-I, and MHC-II with $\mathrm{IC}_{50}$ values.

\begin{tabular}{ccclccc}
\hline S. No & Position & \multicolumn{1}{c}{ Final B-cell Epitope } & MHC-I & IC $_{\mathbf{5 0}}$ & MHC-II $^{\text {IC }}$ \\
\hline 1 & $404-424$ & GDEVRQIAPGQTGKIADYNYK & VRQIAPGQT & 31.27 & VRQIAPGQT & 121 \\
2 & $673-691$ & SYQTQTNSPRRARSVASQS & QTQTNSPRR & 34.53 & QTQTNSPRR & 56 \\
3 & $805-826$ & ILPDPSKPSKRSFIEDLLFNKV & ILPDPSKPS & 23.04 & ILPDPSKPS & 157 \\
4 & $14-36$ & QCVNLTTRTQLPPAYTNSFTRGV & TQLPPAYTN & 11.95 & TQLPPAYTN & 121 \\
\hline
\end{tabular}

Five different constructs were built by the integration of two antigenic peptides and several adjuvants and linkers. There were a lot of possible arrangements of segments but since the analysis of such a huge number of constructs was impossible, we designed these five constructs as shown in Table 2, as samples for our study. The sequence of these constructs differed from each other according to the adjuvant used and also the arrangement order of the constituent segments. The linkers "EAAAK" (blue) links the adjuvant (black) in the constructs. The GGGS and HEYGAEALERAG (grey) link the epitopes. Immunogenic epitopes (red) and the immune enhancer adjuvant, PADRE, as well as linker sequences were inserted as shown in Table 2.

Table 2. Different vaccine constructs (C1-5). An adjuvant is shown in Black, linker 'EAAAK' joining adjuvant shown in Blue, linkers 'GGGS' 'HEYGAEALERAG' joining epitope shown in Grey, epitopes shown in Red, and PADRE sequence shown in Green.

\begin{tabular}{c} 
C1 adjuvant $=$ HBHA adjuvant \\
\hline EAAAKMAENPNIDDLPAPLLAALGAADLALATVNDLIANLRERAEETRAETRTRVEERRARLTKFQEDLPEQF \\
IELRDKFTTEELRKAAEGYLEAATNRYNELVERGAALQRLRSQTAFEDASARAEGYVDQAVELTQEALGTVAS \\
QTRAVGERAAKLVGIELEAAAKAKFVAAWTLKAAAGGSGDEVRQIAPGQTGKIADYNYKGGGSSYTQTN \\
SPRRARSVASQSGGGSAKFVAAWTLKAAAGGGSILPDPSKPSKRSFIEDLLFNKVHEYGAEALERAGQCVNLT \\
TRTQLPPAYTNSFTRGVHEYGAEALERAGAKFVAAWTLKAAAGGGS \\
C2 adjuvant = Beta defensin adjuvant \\
EAAAKGIINTLQKYYCRVRGGRCAVLSCLPKEEQIGKCSTRGRKCCRRKKEAAAKAKFVAAWTLKAAAGGGS \\
GDEVRQIAPGQTGKIADYNYKGGGSILPDPSKPSKRFIEDLLFNKVGGGSAKFVAAWTLKAAAGGGSSYQTQ \\
TNSPRRARSVASQSHEYGAEALERAGQCVNLTTRTQLPPAYTNSFTRGVHEYGAEALERAGAKFVAAWTLKA \\
AAGGGS
\end{tabular}


Table 2. Cont.

\begin{tabular}{l} 
C3 adjuvant = HBHA conserved \\
\hline EAAAKMAENSNIDDIKAPLLAALGAADLALATVNELITNLRERAEETRRSRVEESRARLTKLQEDLPEQLTELR \\
EKFTAEELRKAAEGYLEAATSELVERGEAALERLRSQQSFEEVSARAEGYVDQAVELTQEALGTVASQVEGRAA \\
KLVGIELEAAAKAKFVAATLKAAAGGGSSYTQTNSPRRARSVASQSGGGSQCVLTTTQLPPAYTNSFTR \\
GVGGGSAKFVAAWTLKAAAGGGSGDEVRQIAPGQTGKIADYNYKHEYGAEALERAGILPDPSKPSKRSFIED \\
LLFNKVHEYGAEALERAGAKFVAAWTLKAAAGGGS \\
C4 adjuvant = Ribosomal protein adjuvant \\
\hline EAAAKMAKLSTDELLDAFKEMTLLELSDFVKKFEETFEVTAAAPVAVAAAGAAPAGAAVEAAEEQSEFDVILE \\
AAGDKKIGVIKVVREIVSGLGLKAKDLVDGAPKPLLEKVAKEAADEAKAKLEAAGATVTVKEAAAKAKFVA \\
AWTLKAAAGGGSQCVNLTTRTQLPPAYTNSFTRGVGGGSGDEVRQIAPGQTGKIADYNYKGGGSAKFVAAW \\
TLKAAAGGGSILPDPSKPSKRSFIEDLLFNKVHEYGAEALERAGSYQTQTNSPRRARSVASQSHEYGAEALERA \\
GAKFVAAWTLKAAAGGGS \\
C5 adjuvant = flagellin adjuvant \\
\hline EAAAKMAQVINTNSLSLLTQNNLNKSQSSLSSAIERLSSGLRINSAKDDAAGQAIANRFTSNIKGLTQASRNAN \\
DGISIAQTTEGALNEINNNLQRVRELSVQATNGTNSDSDLKSIQDEIQQRLEEIDRVSNQTQFNGVKVLSQDNQ \\
MKIQVGANDGETITIDLQKIDVKSLGLDGFNVEAAAKAKFVAAWTLKAAAGGGSGDEVRQIAPGQTGKIAD \\
YNYKGGGSSYQTTNSPRARSVASQSGGGSAKFVAAWTLKAAAGGGSILPDPSKPSKRFIEDLLFNKVHEY \\
GAEALERAGQCVNLTTRTQLPPAYTNSFTRGVHEYGAEALERAGAKFVAAWTLKAAAGGGS \\
\hline
\end{tabular}

All the five vaccine constructs were checked for allergenicity, antigenicity, and solubility by Algpred server, Vexijen v2.0 [40] (Designed by Medical University Sofia, Bulgari and The Jenner Institute, Oxford University, Compton, Berkshire, RG20 7NN, UK) and SOLpro, respectively as shown in Table 3. The least allergenic and most antigenic $\mathrm{C} 1$ vaccine construct was selected as a promiscuous vaccine to elicit host immune response.

Table 3. Antigenicity, allergenicity, and solubility of the various vaccine constructs.

\begin{tabular}{cccc}
\hline S. No & $\begin{array}{c}\text { Antigenicity } \\
\text { (Threshold }>\mathbf{0 . 4 )}\end{array}$ & Solubility & $\begin{array}{c}\text { Allergenicity } \\
\text { (Threshold -0.4) }\end{array}$ \\
\hline C1 & 0.4987 & 0.837445 & -0.83923292 \\
C2 & 0.5230 & 0.887539 & -0.75524626 \\
C3 & 0.5147 & 0.858435 & -0.75971333 \\
C4 & 0.4687 & 0.852533 & 0.13431533 \\
C5 & 0.4846 & 0.520147 & 0.51140747 \\
\hline
\end{tabular}

Physiochemical properties of all vaccine constructs were predicted by the ProtParam server as shown in Table 4. The molecular weight of the vaccine constructs was predicted between $23 \mathrm{kDa}$ to $\sim 36 \mathrm{kDa}$. The GRAVY score [41] was found between the values -0.14 to -0.45 , which shows a hydrophilic nature of the vaccine constructs. The high aliphatic index score (64.13 to 80.42) indicates their stability at several temperatures. All vaccines constructs showed good instability index $(<40)$ except construct 3 (C3) and these stable constructs can initiate an immunogenic reaction [42]. The estimated half-life of construct-1 (C-1) is $1 \mathrm{~h}$ in mammalian reticulocytes (in vitro), $30 \mathrm{~min}$ in yeast (in vivo), and more than $10 \mathrm{~h}$ in Escherichia coli (in vivo). Antigenic propensity was calculated for 337 residues of vaccine construct. It was above one for majority residues and considerable variation was seen at some places, with decreased antigenicity near residue 50,175 , and 310 . High span regions with enhanced antigenicity were 13 in total (Figure 3A) and average antigenicity was slightly above 1, i.e., 1.0098. 
Table 4. Physiochemical properties of the vaccine constructs.

\begin{tabular}{ccccccc}
\hline S. No & $\begin{array}{c}\text { Number of } \\
\text { Amino Acids }\end{array}$ & $\begin{array}{c}\text { Molecular } \\
\text { Weight (Daltons) }\end{array}$ & Theoretical pI & $\begin{array}{c}\text { Aliphatic } \\
\text { Index }\end{array}$ & GRAVY & $\begin{array}{c}\text { Instability } \\
\text { Index }\end{array}$ \\
\hline C1 & 337 & $35,906.03$ & 6.00 & 76.44 & -0.431 & 39.46 (stable) \\
C2 & 223 & $23,438.58$ & 9.88 & 64.13 & -0.439 & 36.96 (stable) \\
C3 & 328 & $34,787.80$ & 5.61 & 79.39 & -0.399 & 44.66 (unstable) \\
C4 & 308 & $31,717.87$ & 6.32 & 80.42 & -0.140 & 28.73 (stable) \\
C5 & 353 & $37,181.20$ & 9.01 & 78.39 & -0.451 & 31.65 (stable) \\
\hline
\end{tabular}

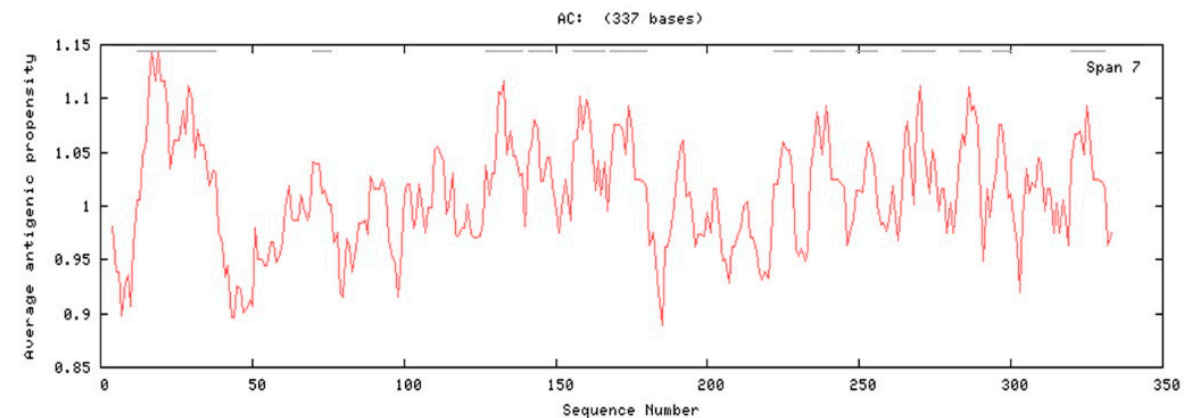

Tue 21 Jul 2020 at 14:01

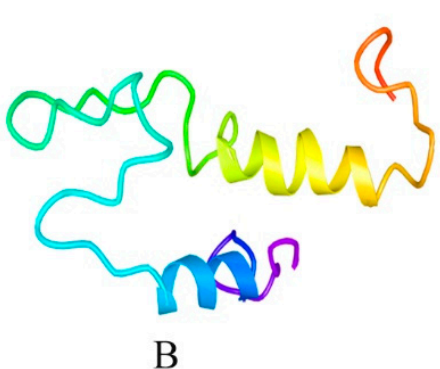

A

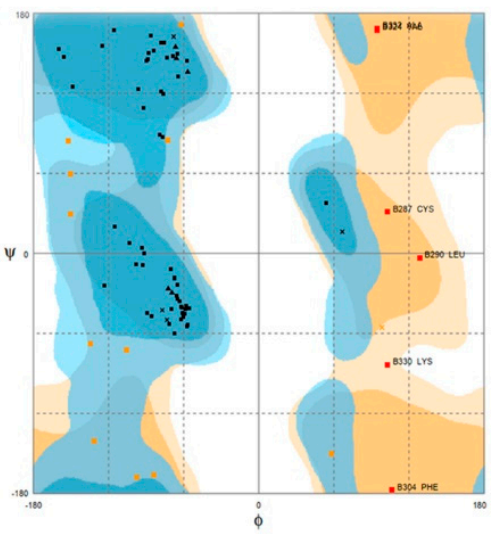

$\mathrm{C}$

Figure 3. (A) The average antigenic propensity (1.0098) of vaccine construct-1 (C-1). Grey lines above the graph show regions with increased antigenicity. (B) 3D structure of the final vaccine, which contains two right-handed alpha helices (DodgerBlue and Yellow) and coil and random coils (DeepSkyBlue, LawnGreen, and OrangeRed). (C) Ramachandran plot of the modeled vaccine construct. The number of residues in favored regions were $63(77.8 \%), 12$ were in allowed $(14.8 \%)$ and $6(7.4 \%)$ were outliers.

These tertiary structures are considered as critical regarding their interactions with other proteins and molecules within the cell. Tertiary structures of the constructed vaccines were generated by using the SWISS-MODEL server. PatchDock, HADDOCK web server, and FireDock were used for molecular docking and refinement, respectively, and then validated by RAMPAGE. The final 3D modeled structure of the vaccine construct is shown in Figure $3 \mathrm{~B}$ and the Ramachandran validation plot is displayed in Figure 3C.

A vaccine able to bind to different HLA allelic proportion of the human population is most important for its proper function inside the host. The docking of all vaccine constructs was performed with TLR7, TLR8, and TLR 4/MD2 complexes (PDB ID 2Z65). After analyzing all vaccine constructs, we finalized the vaccine construct (C-1) based on different physiochemical properties and docking scores. The C-1 TLR7 complex has good global binding energy score $(-65.88 \mathrm{Kcal} / \mathrm{mol})$ obtained from PatchDock as shown in Table 5 . Therefore, we considered construct 1 , as a promising vaccine against the 2019 novel Coronavirus (2019-nCoV). Further molecular docking of the vaccine was performed with the modeled TLR7 using the HADDOCK web server. The docking results revealed that the 
vaccine showed a strong binding affinity with the TLR receptor indicated in Table 6. Molecular interaction formed between vaccine and TLR can be seen in Figure 4, which shows 3 salt bridges, 10 hydrogen bonds, and 156 non bonded interactions between both molecules. Root mean square fluctuation (RMSF) of chain A was observed to be maximum at position 256. This residue was not involved in direct interaction with another chain. However, the nearby residue at position 252 was involved in non-bonded contact formation with Glu at position 769, which means that the interaction had an impact on RMSF. Similar observations were made for chain B where most fluctuations were observed at residue $122,271,354,488$, and near 818 , but these residues were not directly involved in bond formation with chain A. Nearby residue interaction distorts geometry and causes fluctuation of main-chain atom coordinates from alpha carbon backbone.

Table 5. Docking results of TLR4, TLR7, and TLR8 with five vaccine constructs.

\begin{tabular}{ccccccc}
\hline S. No & $\begin{array}{c}\text { Solution } \\
\text { Number }\end{array}$ & $\begin{array}{c}\text { Global Energy } \\
\text { (Kcal/mol) }\end{array}$ & $\begin{array}{c}\text { Attractive } \\
\text { VdW }\end{array}$ & $\begin{array}{c}\text { Repulsive } \\
\text { VdW }\end{array}$ & ACE & HB \\
\hline TLR4/C1 & 268 & -43.48 & -41.07 & 20.13 & 0.39 & -3.21 \\
TLR4/C2 & 250 & -48.43 & -37.87 & 14.87 & 1.01 & -3.09 \\
TLR4/C3 & 753 & -57.79 & -34.68 & 24.66 & -6.82 & -6.15 \\
TLR4/C4 & 58 & -49.06 & -32.34 & 3.16 & 6.95 & -1.79 \\
TLR4/C5 & 307 & -37.22 & -27.03 & 19.07 & -2.02 & -1.64 \\
TLR7/C1 & 94 & -65.88 & -37.52 & 18.56 & -7.02 & -3.37 \\
TLR7/C2 & 438 & -47.16 & -41.24 & 17.91 & 7.72 & -3.18 \\
TLR7/C3 & 256 & -43.91 & -26.80 & 6.60 & 2.39 & -1.62 \\
TLR7/C4 & 839 & -55.98 & -39.32 & 16.45 & -3.15 & -4.69 \\
TLR7/C5 & 620 & -47.34 & -29.54 & 18.96 & -3.22 & -1.92 \\
TLR8/C1 & 383 & -60.24 & -42.98 & 17.59 & -0.09 & -5.04 \\
TLR8/C2 & 273 & -57.44 & -42.20 & 21.45 & 2.39 & -6.75 \\
TLR8/C3 & 973 & -50.57 & -29.17 & 24.59 & -11.45 & -1.43 \\
TLR8/C4 & 280 & -39.20 & -34.23 & 10.77 & 5.27 & -3.89 \\
TLR8/C5 & 902 & -60.34 & -42.13 & 28.02 & 1.06 & -2.06 \\
\hline
\end{tabular}

Table 6. Protein-protein docking results of the vaccine construct and TLR7.

\begin{tabular}{cc}
\hline HADDOCK score & $-132.1+/-7.3$ \\
Cluster size & 45 \\
RMSD from the overall lowest-energy structure & $0.5+/-0.3$ \\
Van der Waals energy & $-117.0+/-6.3$ \\
Electrostatic energy & $-331.2+/-49.7$ \\
Desolvation energy & $-75.8+/-6.4$ \\
Restraints violation energy & $1269.5+/-72.06$ \\
Buried Surface Area & $3552.9+/-125.7$ \\
Z-Score & -2.1 \\
\hline
\end{tabular}

The immune simulation was carried out using C-ImmSim, which accounts for both B-cell and HLA class I/II epitope prediction as well as the interaction of the T-cell receptor with the peptide-HLA complexes. This prediction is made through an agent-based representation of immune cells and the interaction potential of amino acids [43]. Booster doses were given because initial immunization response is comparatively sluggish with low antibody concentration, while the booster immunization is pretty fast, with high-affinity antibody, mostly IgG production in bulk. This is evident for our vaccine construct simulation (Figure 5B), where IgG increased after the booster dose. The positive control showed no change in antibody titer in the absence of the adjuvant. Total count of B lymphocytes and non-memory cells, as well as isotypes IgG1 and IgG2, were varied for C1 vs. control. CD4 T-helper lymphocytes count, especially memory cell count shown in green, is very high in C1 (Figure 6B) compared to control. The concentration of cytokines and interleukins is also considerably increased after $\mathrm{C} 1$ administration (Figure 6D) compared to the control. 


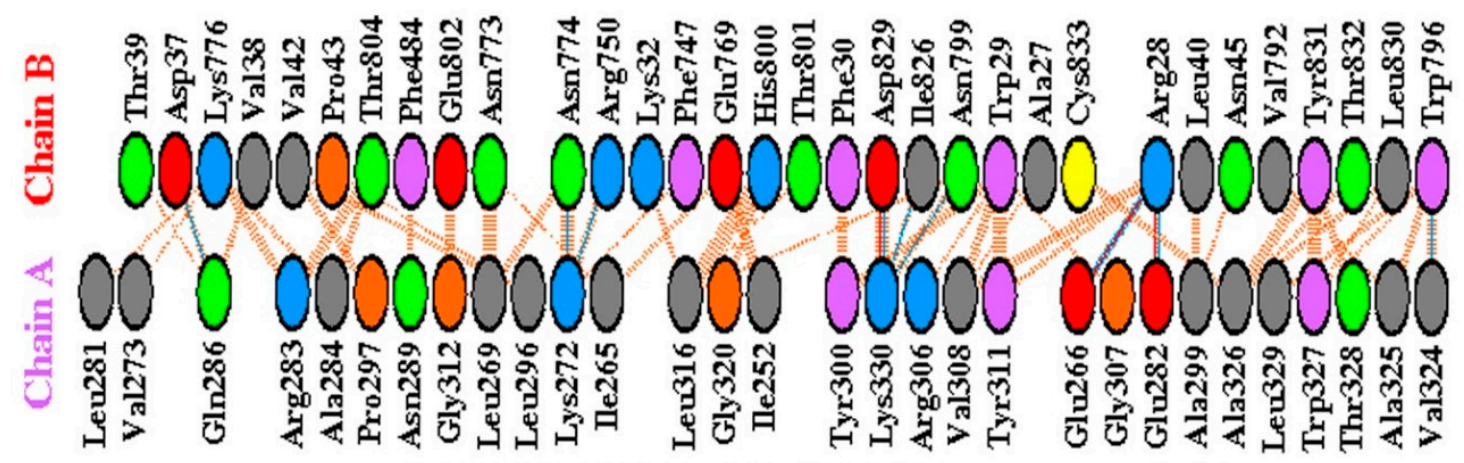

Residue colours: Positive $(H, K, R)$; negative $(D, E) ; S, T, N, Q=$ neutral; $A, V, L, I, M=$ aliphatic; F, Y,W = aromatic; P, G = Pro\&Gly;
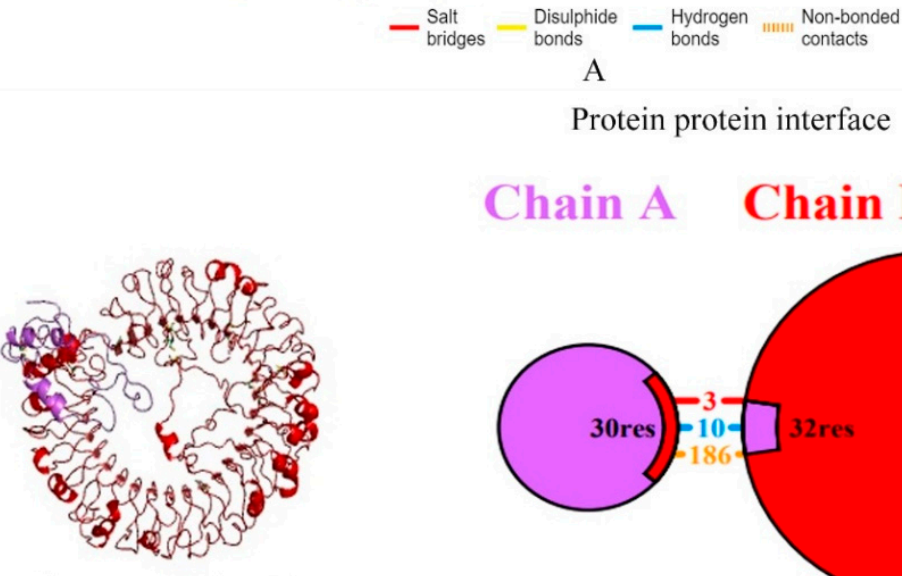

\section{Chain A Chain B}

Chain A and B in 3D

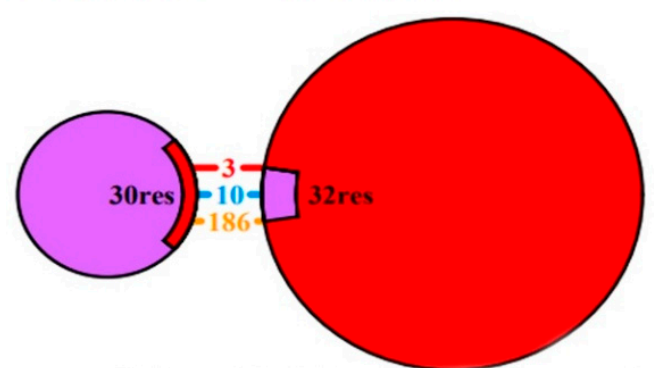

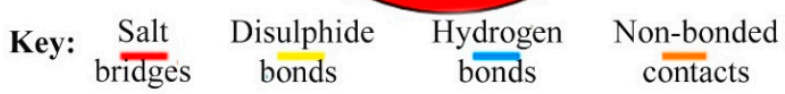

B

Figure 4. (A) The number of $\mathrm{H}$-bond lines between any two residues indicates the number of potential hydrogen bonds between them. For nonbonded contacts, which can be plentiful, the width of the striped line is proportional to the number of atomic contacts. (B) Schematic diagram of interactions between protein chains. Interacting chains are joined by colored lines, each representing a different type of interaction, as per the key above. The area of each circle is proportional to the surface area of the corresponding protein chain. The extent of the interface region on each chain is represented by the black wedge whose size signifies the interface surface area. Chain A refers to a vaccine construct while chain B is toll-like receptor.

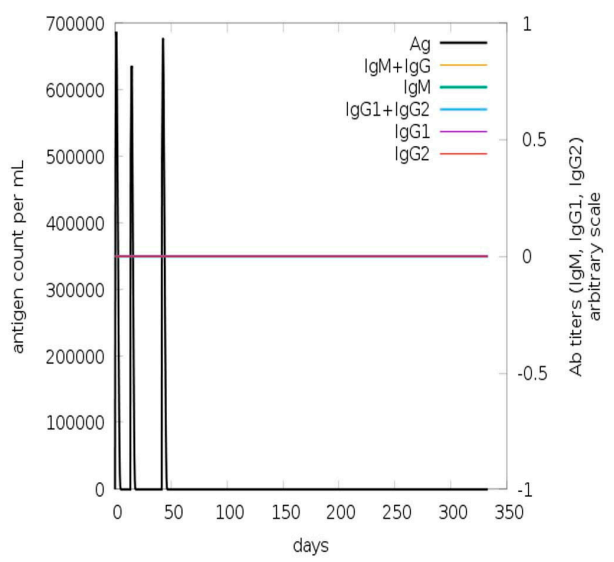

A

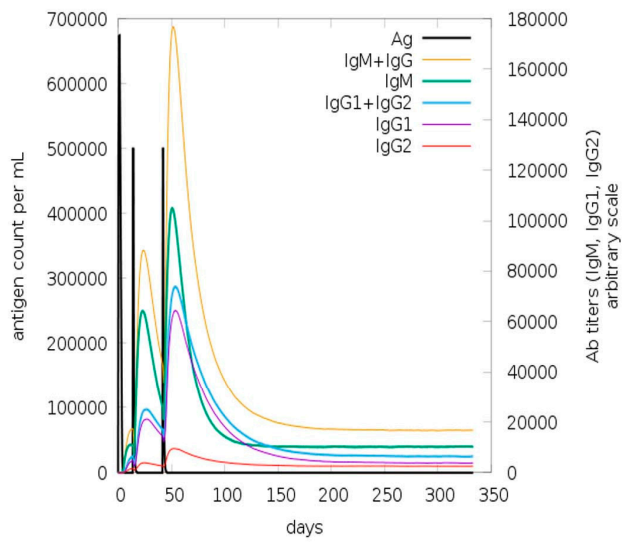

B

Figure 5. Cont. 

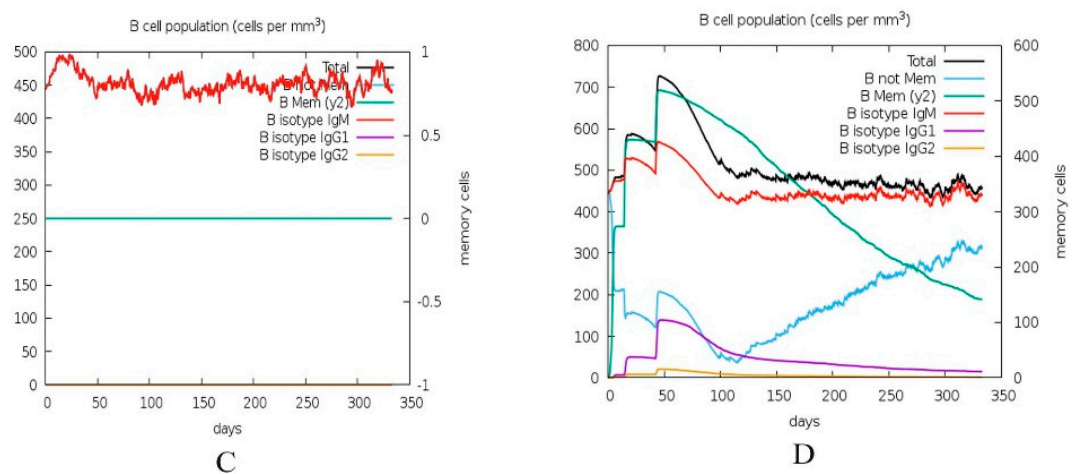

Figure 5. (A) Antigen and immunoglobulins of control, (B) antigen and immunoglobulins of construct C1 with antibodies sub-divided per isotype. (C). B lymphocytes showing total count, memory cells (isotypes IgM, IgG1, and IgG2) for control. (D). B lymphocytes showing total count, memory cells (isotypes $\operatorname{IgM}, \operatorname{IgG1}$, and $\operatorname{IgG} 2$ ) for construct $\mathrm{C} 1$.

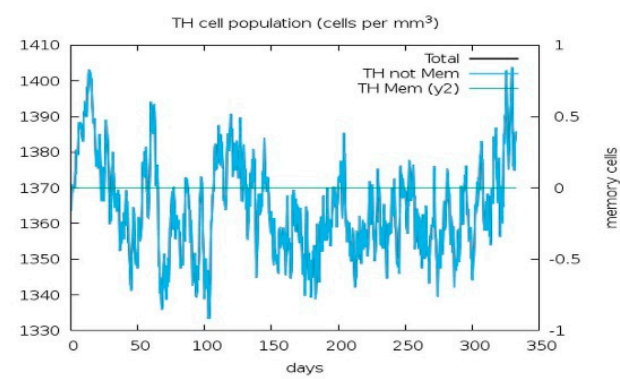

A

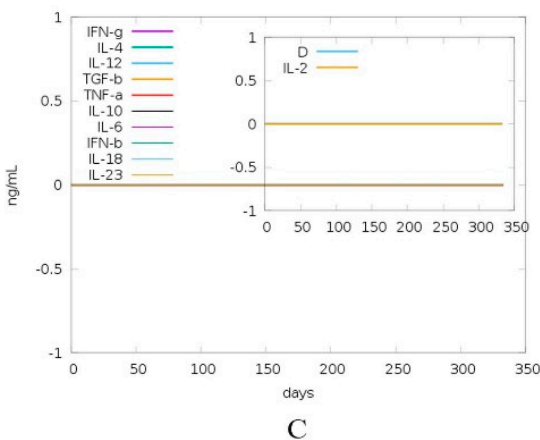

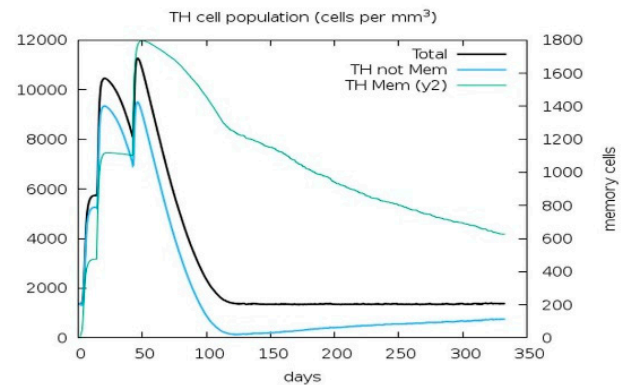

B

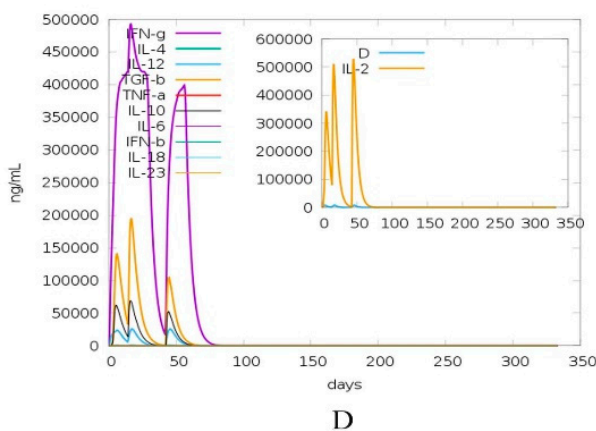

D

Figure 6. (A) The plot shows total and memory counts of CD4 T-helper lymphocytes for control. (B) The plot shows total and memory counts of CD4 T-helper lymphocytes for construct C1. (C). The concentration of cytokines and interleukins for control with $\mathrm{D}$ in the inset plot representing danger signal. (D). The concentration of cytokines and interleukins for construct C1.

To analyze the expression and to clone the constructed vaccine inside a suitable vector, we translate back the protein amino acids sequence to a cDNA nucleotide sequence using the Java codon adaptation tool and EMBOSS Backtranseq. The final vaccine construct showed 54.0\% GC content when analyzed by codon optimization tool. The GC content was in the normal range (30-70\%). The codon optimization index (CAI) value was predicted 1.0, which indicates high expression in E. coli indicated in Figure 7. 


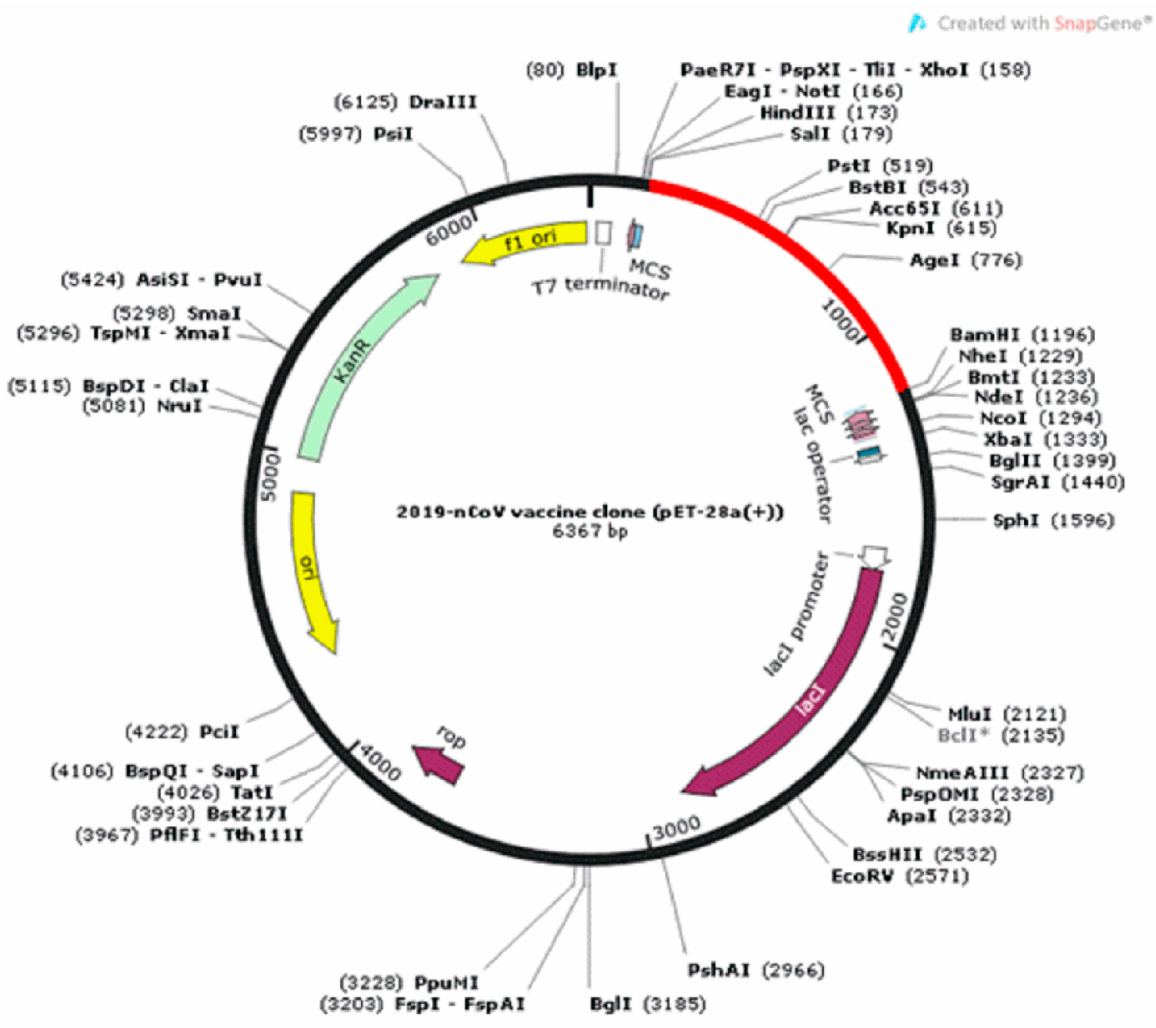

Figure 7. Expression of the final vaccine gene (Red) in a restriction cloning vector pET28a in E. coli host.

\section{Discussion}

Severe acute respiratory syndrome coronavirus 2 (SARS-CoV-2) is a new and challenging virus that causes severe infection in humans. The complete genome sequence of the pathogen was reported by [1] on 29 January 2020. In the present study, we took advantage of the available genomes of SARS-CoV-2 to predict an immunogenic, multi-epitope vaccine with different immune enhancer adjuvants and linker sequences. Different approaches, like an inactivated or weakened virus, replicating or non-replicating viral vector, DNA or RNA and protein-based are used for SARS-CoV-2 vaccine development, as indicated in Figure 8 [44].

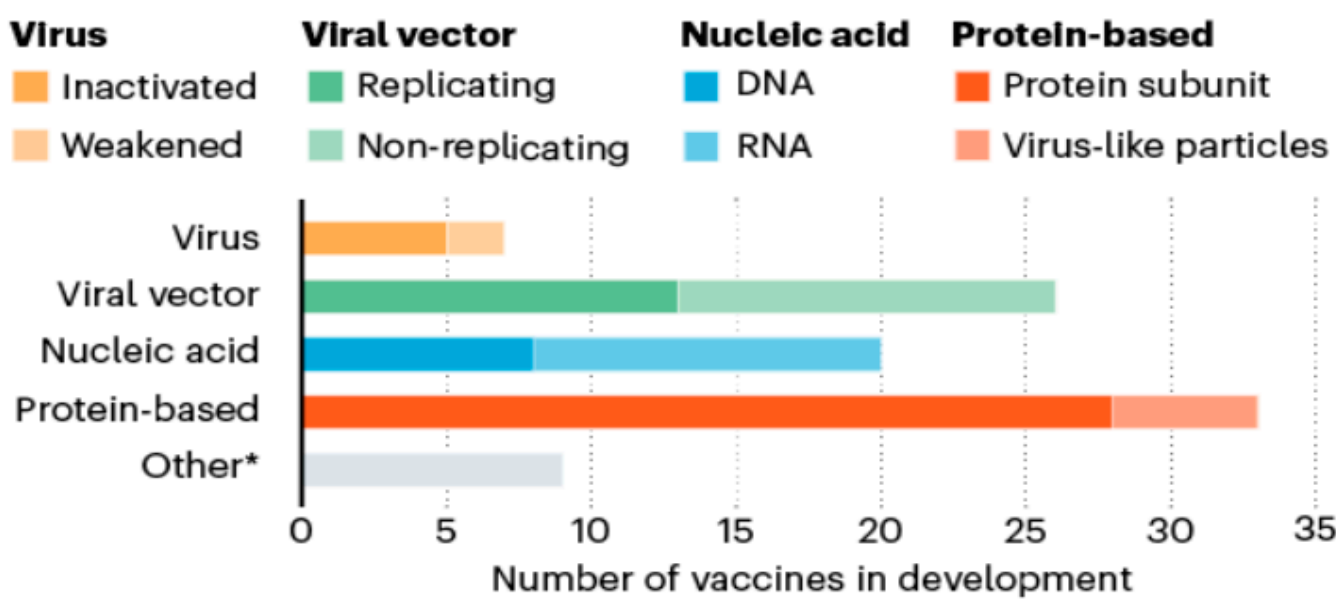

Figure 8. An array of vaccines: eight different approaches including viral-inactivated \& weakened, viral vector-based replicating \& non-replicating, protein-based subunit \& virus-like particles are used for the development of SARS-CoV-2 (source: Nature Briefing). 
Twenty-eight teams are working on vaccines with viral protein subunits, most of these groups are focusing on spike protein. We also selected spike protein to develop potent vaccine construct from the different immunogenic determinant of the spike glycoprotein of SARS-CoV-2. The immunogenic, multi-epitope, subunit vaccine was generated from MHC-I, MHC-II alleles, B-Cell and IFN-inducing epitopes of the surface glycoprotein (S) [16]. Only peptides having IC50 values $<200$ were considered as effective peptides. All the selected epitopes were merged with different adjuvants, linkers, and Pan-DR sequence epitopes (PADRE). PADRE sequence is responsible for the reduction in polymorphism in HLA DR molecules in the population [45]. We have also used the G-rich linker GGGS which enhances the immunogenicity of the vaccine inside the host [46]. Five vaccine constructs $(C 1, C 2, C 3, C 4, C 5)$ were made. All the vaccine constructs were further analyzed for antigenicity, solubility, and allergenicity. We also predicted the physicochemical properties of vaccines and shortlisted the one vaccine construct, C1, which showed good properties among others with 1.0093 average antigenic propensity as shown in Figure 3. As the molecular weight of the candidate vaccine $\mathrm{C} 1$ is $35.9 \mathrm{kDa}$, this makes it possible to predict its solubility during expression, such a molecular weight being able to trigger an immune response. The theoretical pI [47] is predicted to be 6.0, indicating that the protein is acidic. In addition, the predicted instability index [48] indicates that the vaccine peptide will be stable upon expression, thus further firming its potential for use. The aliphatic index indicates good hydrophobicity [41,47]. All the above properties support our constructed vaccine as promiscuous against the SARS-CoV-2. The 3D model of the construct was built with the online server and validated by Ramachandran plot analysis. Furthermore, the docking analysis of the final vaccine $\mathrm{C} 1$ was done with different toll-like receptors (TLR4, TLR7, and TLR8). TLR4 has been involved in the recognition of viral structural and non-structural proteins leading to inflammatory cytokine production [49]. TLR4-activating viral proteins include the RSV fusion protein (F), the EBOV glycoprotein, the vesicular stomatitis virus glycoprotein (VSV G), and the dengue virus (DENV) [50]. TLR7 and 8 are key players in antiviral responses. TLR7-specific agonists activate plasmacytoid dendritic cells (pDCs) and B cells and mainly induce IFN- $\alpha$ and IFN-regulated cytokines. TLR8-specific agonists activate myeloid DCs, monocytes, and monocyte-derived DC, leading primarily to the production of proinflammatory cytokines and chemokines, such as TNF- $\alpha$, IL-12, and MIP-1 $\alpha$ [51]. The ability of TLR7 and TLR8 agonists to activate DCs and thus elicit Th1 and CD8+ T cell responses can be exploited to enhance the efficacy of vaccination [52,53]. Activation of TLR7/8 triggers different signaling pathways in human monocytes that halt viral pathogenesis by the induction of interferons (TFNs). In the innate immune system, plasmacytoid dendritic cells TLR7 and TLR9 trigger induction of proinflammatory cytokines and IFN- $\alpha / \beta$. The docking results showed good global energy scores for TLR7 that we used during the analysis for the vaccine $\mathrm{C} 1$, which was the indication of eliciting an optimal immune response against the SARS-CoV-2. The docked complex when simulated using CABS-flex (2) dynamics revealed that both molecules remained stable with minimum fluctuations RMSF. The flexibility of both molecules can be seen in the trajectory in Figure 9.

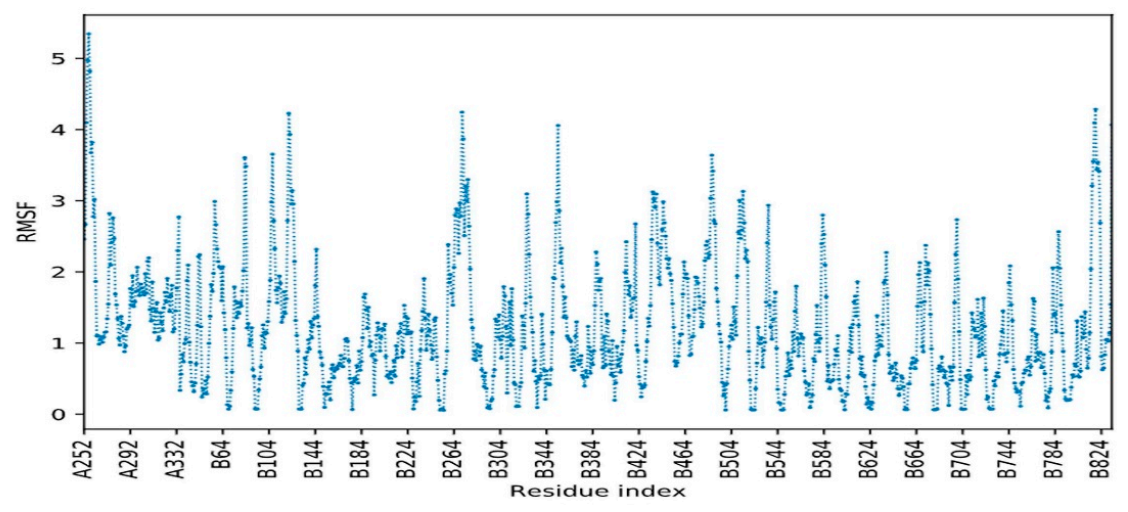

Figure 9. RMSF of chain A (vaccine construct) and B (TLR receptor) of the docked complex. Residue starting with A belong to chain A, while those starting with B reflect chain B residues. 
Simulation by C-ImmSim through a virtual injection was done for HLA allele-specific (HLA-A*0101, HLA-A*0201, HLA-B*0702, HLA-B*3901, HLA-DRB1*0101, and HLA-DRB1*0401) outcome of the vaccine construct. This was to check the proficiency of construct to the adaptive immune system (reliant on T-cell receptor diversity, through $\mathrm{V}, \mathrm{J}$ arrangements of the alpha and $\mathrm{V}, \mathrm{D}, \mathrm{J}$ arrangements of the beta unit in the thymus). The output of the simulator was a graphical illustration of the total count of lymphocytes, division amid isotypes, antibody, and cytokine concentration. Cells may bind or move and follow environmental harmonized behavior [43,54]. The decrease in antigen count was observed for $\mathrm{C} 1$ after 50 days and diversity finally reached zero around the 70th day of injection (Figure 6D). The antibodies titers (IgM and IgG1 + IgG2) showed a high peak after booster doses of C1 injection. Greater IgM production is required for enhanced primary immune response, resulting in augmentation of $\mathrm{B}$ cell population and additional antibodies responsible for secondary and tertiary immune reactions [12]. Overall B cell population was found highest around the 50th day (in cells per $\mathrm{mm}^{3}$ ) of injecting $\mathrm{C} 1$, before plateauing off. Increased CD4 T-helper cell population has a vital role in evoking protection and was evoked after injection (Figure 6B). Nevertheless, this prediction is preliminary and the study of the construct with diverse HLA-alleles is suggested as antigen-specific immune response relies not only on age, dose, the time interval of booster dose, but mostly immunogenetics of population.

Vaccine construct $\mathrm{C} 1$ was cloned in silico with the help of Snapgene in the most suitable plasmid vector pET28a (+) by restriction enzymes SalI and BamHI to check its expression and purification in the bacterial cellular environment. Analysis of the virtual cloning, after codon optimization, validated the stance that translated chimeric vaccine construct appears proficient with enhanced gene expression and is capable of vaccine production at an economical cost.

\section{Conclusions}

In conclusion, we utilized the immunogenic B-cell, T-cell, and IFN-inducing epitopes to generate a peptide-based multi-epitope vaccine from the surface glycoprotein which elicits humoral and cell-mediated immunity, respectively, to eradicate viral particles. Predicted epitopes were merged using appropriate linkers and adjuvants to enhance the immunogenicity of the vaccine. Antigenicity, allergenicity, and solubility, as well as physiochemical properties and tertiary structure analysis, were confirmed. Docking and MD simulation analysis of TLR7 and vaccine were performed, allowing evaluation of the binding affinity and stability of the complex. The immune simulation showed an enhanced antibody titer production and CD4 T-cell count after $\mathrm{C} 1$ virtual administration. It also shows that adjuvant has an important role in healthy immune response elicitation. The final vaccine construct was back-translated and in silico cloned in a plasmid, which showed effective expression.

Author Contributions: N.R., F.A. and M.S. performed the analysis and prepared the initial draft. Z.B. performed M.D. and immune system simulation, reviewed for critical analysis and helped draft final version of manuscript while M.K.K., P.J., E.N. and K.K. designed the study. H.K. finalized the draft and supervised the overall study. All authors have read and agreed to the published version of the manuscript.

Funding: It was supported by the University of Hradec Kralove (Faculty of Science, VT2019-2021).

Conflicts of Interest: The authors declare that they have no conflict of interest.

\section{References}

1. Lu, R.; Zhao, X.; Li, J.; Niu, P.; Yang, B.; Wu, H.; Wang, W.; Song, H.; Huang, B.; Zhu, N.; et al. Articles Genomic characterisation and epidemiology of 2019 novel coronavirus: Implications for virus origins and receptor binding. Lancet 2020, 6736, 1-10. [CrossRef]

2. Alejandra Tortorici, M.; Walls, A.C.; Lang, Y.; Wang, C.; Li, Z.; Koerhuis, D.; Boons, G.J.; Bosch, B.J.; Rey, F.A.; de Groot, R.J.; et al. Structural basis for human coronavirus attachment to sialic acid receptors. Nat. Struct. Mol. Biol. 2019, 26, 481-489. [CrossRef] [PubMed]

3. Lu, G.; Wang, Q.; Gao, G.F. Bat-to-human: Spike features determining "host jump" of coronaviruses SARS-CoV, MERS-CoV, and beyond. Trends Microbiol. 2015, 23, 468-478. [CrossRef] [PubMed] 
4. Ge, X.Y.; Li, J.L.; Yang, X.-L.; Chmura, A.A.; Zhu, G.; Epstein, J.H.; Mazet, J.K.; Hu, B.; Zhang, W.; Peng, C.; et al. Isolation and characterization of a bat SARS-like coronavirus that uses the ACE2 receptor. Nature 2013, 503, 535-538. [CrossRef] [PubMed]

5. Haagmans, B.L.; Al Dhahiry, S.H.S.; Reusken, C.B.E.M.; Raj, V.S.; Galiano, M.; Myers, R.; Godeke, G.J.; Jonges, M.; Farag, E.; Diab, A.; et al. Middle East respiratory syndrome coronavirus in dromedary camels: An outbreak investigation. Lancet Infect. Dis. 2014, 14, 140-145. [CrossRef]

6. Su, S.; Wong, G.; Shi, W.; Liu, J.; Lai, A.C.K.; Zhou, J.; Liu, W.; Bi, Y.; Gao, G.F. Epidemiology, Genetic Recombination, and Pathogenesis of Coronaviruses. Trends Microbiol. 2016, 24, 490-502. [CrossRef]

7. Walls, A.C.; Park, Y.J.; Tortorici, M.A.; Wall, A.; McGuire, A.T.; Veesler, D. Structure, Function, and Antigenicity of the SARS-CoV-2 Spike Glycoprotein. Cell 2020, 181, 281-292.e6. [CrossRef]

8. Zheng, M.; Song, L. Novel antibody epitopes dominate the antigenicity of spike glycoprotein in SARS-CoV-2 compared to SARS-CoV. Cell. Mol. Immunol. 2020, 17, 536-538. [CrossRef]

9. Yuan, M.; Wu, N.C.; Zhu, X.; Lee, C.C.D.; So, R.T.Y.; Lv, H.; Mok, C.K.P.; Wilson, I.A. A highly conserved cryptic epitope in the receptor binding domains of SARS-CoV-2 and SARS-CoV. Science 2020, 368, 630-633. [CrossRef]

10. Rahman, N.; Basharat, Z.; Yousuf, M.; Castaldo, G.; Rastrelli, L. Virtual Screening of Natural Products Against Type II Transmembrane Serine Protease (TMPRSS2), the Priming Agent of Coronavirus 2 (SARS-CoV-2). Molecules 2020, 25, 2271. [CrossRef]

11. Li, Z.; Tomlinson, A.C.A.; Wong, A.H.M.; Zhou, D.; Desforges, M.; Talbot, P.J.; Benlekbir, S.; Rubinstein, J.L.; Rini, J.M. The human coronavirus HCoV-229E S-protein structure and receptor binding. Elife 2019, 8, 1-22. [CrossRef]

12. Walls, A.C.; Tortorici, M.A.; Bosch, B.J.; Frenz, B.; Rottier, P.J.M.; DiMaio, F.; Rey, F.A.; Veesler, D. Cryo-electron microscopy structure of a coronavirus spike glycoprotein trimer. Nature 2016, 531, 114-117. [CrossRef] [PubMed]

13. Shey, R.A.; Ghogomu, S.M.; Esoh, K.K.; Nebangwa, N.D.; Shintouo, C.M.; Nongley, N.F.; Asa, B.F.; Ngale, F.N.; Vanhamme, L.; Souopgui, J. In-silico design of a multi-epitope vaccine candidate against onchocerciasis and related filarial diseases. Sci. Rep. 2019, 9, 1-18. [CrossRef] [PubMed]

14. Fleri, W.; Paul, S.; Dhanda, S.K.; Mahajan, S.; Xu, X.; Peters, B.; Sette, A. The immune epitope database and analysis resource in epitope discovery and synthetic vaccine design. Front. Immunol. 2017, 8, 278. [CrossRef] [PubMed]

15. Wang, P.; Sidney, J.; Dow, C.; Mothé, B.; Sette, A.; Peters, B. A Systematic Assessment of MHC Class II Peptide Binding Predictions and Evaluation of a Consensus Approach. PLoS Comput. Biol. 2008, 4, e1000048. [CrossRef]

16. Kim, Y.; Ponomarenko, J.; Zhu, Z.; Tamang, D.; Wang, P.; Greenbaum, J.; Lundegaard, C.; Sette, A.; Lund, O.; Bourne, P.E.; et al. Immune epitope database analysis resource. Nucleic Acids Res. 2012, 40, W525-W530. [CrossRef]

17. Rahman, N.; Ajmal, A.; Ali, F.; Rastrelli, L. Core proteome mediated therapeutic target mining and multi-epitope vaccine design for Helicobacter pylori. Genomics 2020. [CrossRef]

18. El-manzalawy, Y.; Dobbs, D.; Honavar, V. Predicting linear B-cell epitopes using string kernels. J. Mol. Recognit. Interdiscip. J. 2008, 21, 243-255. [CrossRef]

19. Jespersen, M.C.; Peters, B.; Nielsen, M.; Marcatili, P. BepiPred-2.0: Improving sequence-based B-cell epitope prediction using conformational epitopes. Nucleic Acids Res. 2017, 45, W24-W29. [CrossRef]

20. Hajighahramani, N.; Nezafat, N.; Eslami, M.; Negahdaripour, M.; Rahmatabadi, S.S.; Ghasemi, Y. Immunoinformatics analysis and in silico designing of a novel multi-epitope peptide vaccine against Staphylococcus aureus. Infect. Genet. Evol. 2017, 48, 83-94. [CrossRef]

21. Ahmad, T.A.; Eweida, A.E.; Sheweita, S.A. B-cell epitope mapping for the design of vaccines and effective diagnostics. Trials Vaccinol. 2016, 5, 71-83. [CrossRef]

22. Rosa, D.S.; Tzelepis, F.; Cunha, M.G.; Soares, I.S.; Rodrigues, M.M. The pan HLA DR-binding epitope improves adjuvant-assisted immunization with a recombinant protein containing a malaria vaccine candidate. Immunol. Lett. 2004, 92, 259-268. [CrossRef] [PubMed]

23. Jung, D.; Jeong, S.K.; Lee, C.M.; Noh, K.T.; Heo, D.R.; Shin, Y.K.; Yun, C.H.; Koh, W.J.; Akira, S.; Whang, J.; et al. Enhanced efficacy of therapeutic cancer vaccines produced by Co-treatment with mycobacterium tuberculosis heparin-binding hemagglutinin, a novel TLR4 agonist. Cancer Res. 2011, 71, 2858-2870. [CrossRef] [PubMed] 
24. Ferris, L.K.; Mburu, Y.K.; Mathers, A.R.; Fluharty, E.R.; Larregina, A.T.; Ferris, R.L.; Falo, L.D. Human beta-defensin 3 induces maturation of human langerhans cell-like dendritic cells: An antimicrobial peptide that functions as an endogenous adjuvant. J. Invest. Dermatol. 2013, 133, 460-468. [CrossRef] [PubMed]

25. Park, H.J.; Jang, G.Y.; Kim, Y.S.; Park, J.H.; Lee, S.E.; Vo, M.C.; Lee, J.J.; Han, H.D.; Jung, I.D.; Kang, T.H.; et al. A novel TLR4 binding protein, 40 S ribosomal protein S3, has potential utility as an adjuvant in a dendritic cell-based vaccine. J. Immunother. Cancer 2019, 7, 1-13. [CrossRef] [PubMed]

26. Mizel, S.B.; Bates, J.T. Flagellin as an Adjuvant: Cellular Mechanisms and Potential. J. Immunol. 2010, 185, 5677-5682. [CrossRef]

27. Gnjatic, S.; Sawhney, N.B.; Bhardwaj, N. Toll-Like Receptor Agonists. Cancer J. 2010, 16, 382-391. [CrossRef]

28. Nezafat, N.; Ghasemi, Y.; Javadi, G.; Khoshnoud, M.J.; Omidinia, E. A novel multi-epitope peptide vaccine against cancer: An in silico approach. J. Theor. Biol. 2014, 349, 121-134. [CrossRef]

29. Solanki, V.; Tiwari, V. Subtractive proteomics to identify novel drug targets and reverse vaccinology for the development of chimeric vaccine against Acinetobacter baumannii. Sci. Rep. 2018, 8. [CrossRef]

30. Doytchinova, I.; Flower, D.R. Bioinformatic approach for identifying parasite and fungal candidate subunit vaccines. Open Vaccine J. 2008, 1, 4. [CrossRef]

31. Saha, S.; Raghava, G.P.S. AlgPred: Prediction of allergenic proteins and mapping of IgE epitopes. Nucleic Acids Res. 2006, 34, W202-W209. [CrossRef] [PubMed]

32. Magnan, C.N.; Randall, A.; Baldi, P. SOLpro: Accurate sequence-based prediction of protein solubility. Bioinformatics 2009, 25, 2200-2207. [CrossRef] [PubMed]

33. Gasteiger, E.; Gattiker, A.; Hoogland, C.; Ivanyi, I.; Appel, R.D.; Bairoch, A. ExPASy: The proteomics server for in-depth protein knowledge and analysis. Nucleic Acids Res. 2003, 31, 3784-3788. [CrossRef]

34. Modelos, C. Trabajo práctico N 13. Varianzas en función de variable independiente categórica. Nat. Protoc. 2016, 10, 845-858.

35. Rahman, N.; Muhammad, I.; Nayab, G.E.; Khan, H.; Aschner, M.; Filosa, R.; Daglia, M. Molecular Docking of Isolated Alkaloids for Possible $\alpha$-Glucosidase Inhibition. Biomolecules 2019, 9, 544. [CrossRef]

36. Muhammad, I.; Rahman, N.; Nayab, G.E.; Niaz, S.; Shah, M.; Afridi, S.G.; Khan, H.; Daglia, M.; Capanoglu, E. The Molecular Docking of Flavonoids Isolated from Daucus carota as a Dual Inhibitor of MDM2 and MDMX. Recent Pat. Anticancer. Drug Discov. 2020, 15, 1-11. [CrossRef] [PubMed]

37. Mashiach, E.; Schneidman-Duhovny, D.; Andrusier, N.; Nussinov, R.; Wolfson, H.J. FireDock: A web server for fast interaction refinement in molecular docking. Nucleic Acids Res. 2008, 36, 229-232. [CrossRef]

38. Kaba, S.A.; Karch, C.P.; Seth, L.; Ferlez, K.M.B.; Storme, C.K.; Pesavento, D.M.; Laughlin, P.Y.; Bergmann-Leitner, E.S.; Burkhard, P.; Lanar, D.E. Self-assembling protein nanoparticles with built-in flagellin domains increases protective efficacy of a Plasmodium falciparum based vaccine. Vaccine 2018, 36, 906-914. [CrossRef]

39. Rapin, N.; Lund, O.; Bernaschi, M.; Castiglione, F. Computational immunology meets bioinformatics: The use of prediction tools for molecular binding in the simulation of the immune system. PLOS ONE 2010, 5. [CrossRef]

40. Grote, A.; Hiller, K.; Scheer, M.; Münch, R.; Nörtemann, B.; Hempel, D.C.; Jahn, D. JCat: A novel tool to adapt codon usage of a target gene to its potential expression host. Nucleic Acids Res. 2005, 33, 526-531. [CrossRef]

41. Kyte, J.; Doolittle, R.F.; Diego, S.; Jolla, L. A Simple Method for Displaying the Hydropathic Character of a Protein. J. Mol. Biol. 1982, 157, 105-132. [CrossRef]

42. Wilkins, M.R.; Gasteiger, E.; Bairoch, A.; Sanchez, J.; Williams, K.L.; Appel, R.D.; Hochstrasser, D.F. Protein Identification and Analysis Tools in the ExPASy Server. In The Proteomics Protocols Handbook; Humana Press: Totowa, NJ, USA, 2005; Volume 112, pp. 531-552.

43. Mohan, R.; Venugopal, S. Computational structural and functional analysis of hypothetical proteins of Staphylococcus aureus. Bioinformation 2012, 8, 722. [CrossRef] [PubMed]

44. Miyazawa, S.; Jernigan, R.L. Residue-residue potentials with a favorable contact pair term and an unfavorable high packing density term, for simulation and threading. J. Mol. Biol. 1996, 256, 623-644. [CrossRef]

45. Thanh Le, T.; Andreadakis, Z.; Kumar, A.; Gómez Román, R.; Tollefsen, S.; Saville, M.; Mayhew, S. The COVID-19 vaccine development landscape. Nat. Rev. Drug Discov. 2020, 1-7. [CrossRef]

46. Ghaffari-Nazari, H.; Tavakkol-Afshari, J.; Jaafari, M.R.; Tahaghoghi-Hajghorbani, S.; Masoumi, E.; Jalali, S.A. Improving multi-epitope long peptide vaccine potency by using a strategy that enhances CD4+ T Help in BALB/c mice. PLoS ONE 2015, 10. [CrossRef] 
47. Yang, Y.; Sun, W.; Guo, J.; Zhao, G.; Sun, S.; Yu, H.; Guo, Y.; Li, J.; Jin, X.; Du, L.; et al. In silico design of a DNA-based HIV-1 multi-epitope vaccine for Chinese populations. Hum. Vaccines Immunother. 2015, 11, 795-805. [CrossRef] [PubMed]

48. Swati, S.; Ashok, S. Bioinformatics approaches for structural and functional analysis of proteins in secondary metabolism in Withania somnifera. Mol. Biol. Rep. 2014. [CrossRef]

49. Guruprasad, K.; Reddy, B.V.B.; Pandit, M.W. Correlation between stability of a protein and its dipeptide composition: A novel approach for predicting in vivo stability of a protein from its primary sequence. Protein Eng. Des. Sel. 1990, 4, 155-161. [CrossRef] [PubMed]

50. Lester, S.N.; Li, K. Toll-like receptors in antiviral innate immunity. J. Mol. Biol. 2014, 426, 1246-1264. [CrossRef]

51. Olejnik, J.; Hume, A.J.; Mühlberger, E. Toll-like receptor 4 in acute viral infection: Too much of a good thing. PLoS Pathog. 2018, 14. [CrossRef]

52. Gorden, K.B.; Gorski, K.S.; Gibson, S.J.; Kedl, R.M.; Kieper, W.C.; Qiu, X.; Tomai, M.A.; Alkan, S.S.; Vasilakos, J.P. Synthetic TLR Agonists Reveal Functional Differences between Human TLR7 and TLR8. J. Immunol. 2005, 174, 1259-1268. [CrossRef] [PubMed]

53. Craft, N.; Bruhn, K.W.; Nguyen, B.D.; Prins, R.; Lin, J.W.; Liau, L.M.; Miller, J.F. The TLR7 Agonist Imiquimod Enhances the Anti-Melanoma Effects of a Recombinant Listeria monocytogenes Vaccine. J. Immunol. 2005, 175, 1983-1990. [CrossRef] [PubMed]

54. Wille-Reece, U.; Flynn, B.J.; Loré, K.; Koup, R.A.; Kedl, R.M.; Mattapallil, J.J.; Weiss, W.R.; Roederer, M.; Seder, R.A. HIV Gag protein conjugated to a Toll-like receptor 7/8 agonist improves the magnitude and quality of Th1 and CD8+ T cell responses in nonhuman primates. Proc. Natl. Acad. Sci. USA 2005, 102, 15190-15194. [CrossRef] [PubMed]

(C) 2020 by the authors. Licensee MDPI, Basel, Switzerland. This article is an open access article distributed under the terms and conditions of the Creative Commons Attribution (CC BY) license (http://creativecommons.org/licenses/by/4.0/). 